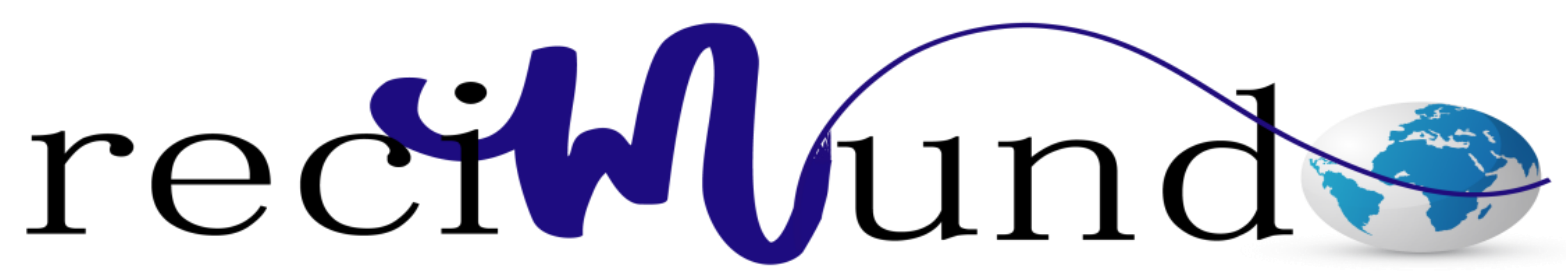

Revista Cientifica Mundo de la Investigación y el Conocimiento

\author{
María Soledad Novillo Valdivieso a; Irina María Ayala López ${ }^{\text {b; }}$ Marlith Gabriela \\ Mora Gonzalez $^{c}$; Leandro Olivero Hurtado Herdoiza ${ }^{d}$
}

Diagnóstico y tratamiento de la nefritis lúpica

Diagnosis and treatment of lupus nephritis

Revista Científica Mundo de la Investigación y el Conocimiento. Vol. 3 núm.3, septiembre, ISSN: 2588-073X, 2019, pp. 410-427

DOI: 10.26820/recimundo/3.(3).septiembre.2019.410-427

URL: http://recimundo.com/index.php/es/article/view/528

Código UNESCO: 3205 Medicina Interna

Tipo de Investigación: Artículo de Revisión

Editorial Saberes del Conocimiento

Recibido: 15/05/2019

Aceptado: 23/06/2019

Publicado: 30/09/2019

Correspondencia: mari_sole24@hotmail.com

a. Médico General; Investigador Independiente; Guayaquil, Ecuador; mari sole24@ hotmail.com
b. Médico; Investigador Independiente; Guayaquil, Ecuador; iri_aylo75@ hotmail.com
c. Médico; Investigador Independiente; Guayaquil, Ecuador; mmarlith@yahoo.es
d. Médico; Investigador Independiente; Guayaquil, Ecuador; leandrooliverh1@ @mail.com 


\section{Diagnóstico y tratamiento de la nefritis lúpica}

Vol. 3, núm. 3., (2019)

María Soledad Novillo Valdivieso; Irina María Ayala López; Marlith Gabriela Mora Gonzalez; Leandro Olivero Hurtado Herdoiza

\section{RESUMEN}

La nefritis lúpica (LN) es una causa significativa de morbilidad y mortalidad en pacientes con lupus eritematoso sistémico. El reconocimiento y diagnóstico de LN retardada pueden ser una causa común de enfermedad renal crónica entre los pacientes. La biopsia renal es el estándar de oro para el diagnóstico de LN; sin embargo, este servicio no está disponible en muchos centros y el uso de análisis de orina, el examen microscópico de la orina y otras pruebas serológicas pueden ser útiles en la identificación de pacientes con LN proliferativa. Los tipos proliferativos de LN (clase III, de clase IV y clase V mixta) comprenden la mayor proporción de pacientes con esta condición. Los pacientes que reciben terapia inmunosupresora deben ser supervisados de cerca para los efectos secundarios y toxicidades relacionadas con las drogas. Los pacientes de LN con enfermedad renal en etapa terminal (clase VI) necesitan estar preparados para la terapia de reemplazo renal (diálisis y el trasplante renal). En todos los pacientes, el tratamiento debe incluir terapias adyuvantes tales como renina angiotensina aldosterona bloqueo del sistema, la protección ósea (con suplementos de calcio y vitamina D), el control de la presión arterial y la cloroquina - todos los cuales ayudan a retardar la progresión de la enfermedad renal.

Palabras Claves: Enfermedad renal; Nefritis lúpica; Diagnóstico; Tratamiento. 


\title{
Diagnóstico y tratamiento de la nefritis lúpica
}

Vol. 3, núm. 3., (2019)

María Soledad Novillo Valdivieso; Irina María Ayala López; Marlith Gabriela Mora Gonzalez; Leandro Olivero Hurtado Herdoiza

\begin{abstract}
Lupus nephritis (LN) is a significant cause of morbidity and mortality in patients with systemic lupus erythematosus. The recognition and diagnosis of delayed LN can be a common cause of chronic kidney disease among patients. Renal biopsy is the gold standard for the diagnosis of LN; However, this service is not available in many centers and the use of urine analysis, microscopic examination of urine and other serological tests may be useful in the identification of patients with proliferative LN. The proliferative types of LN (class III, class IV and mixed class V) comprise the highest proportion of patients with this condition. Patients receiving immunosuppressive therapy should be closely monitored for side effects and drug-related toxicities. LN patients with end-stage renal disease (class VI) need to be prepared for renal replacement therapy (dialysis and renal transplantation). In all patients, treatment should include adjuvant therapies such as renin angiotensin aldosterone system blockage, bone protection (with calcium and vitamin D supplements), blood pressure control and chloroquine - all of which help delay progression of kidney disease.
\end{abstract}

Key Words: Kidney disease; Lupus nephritis; Diagnosis; Treatment.

\section{Introducción.}




\section{Diagnóstico y tratamiento de la nefritis lúpica}

Vol. 3, núm. 3., (2019)

María Soledad Novillo Valdivieso; Irina María Ayala López; Marlith Gabriela Mora Gonzalez; Leandro Olivero Hurtado Herdoiza

El lupus eritematoso sistémico (LES) es un trastorno autoinmune multisistémica crónica con una dilección pre para las mujeres jóvenes. La enfermedad renal en el LES (También conocida como lupus nefritis $(\mathrm{LN})$ ) es una manifestación común de LES y constituye un importante factor pronóstico de estos pacientes. Hasta el 50\% de los pacientes con LES tienen anomalías de la función renal o en la orina (proteinuria, hematuria o cilindros celulares) temprano en el curso de la enfermedad, mientras que aproximadamente el $80 \%$ puede más tarde desarrollar alteraciones evidentes de la función renal (Cameron, 1999). De acuerdo con los criterios de 2012 Systemic Lupus International que colaboran con clasificaciones Clínicas (SLICC) para el diagnóstico de LES, la enfermedad renal está presente cuando un paciente con LES se presenta con proteinuria persistente (>0,5 g / 24 h) o de glóbulos rojos celular yesos. LN es un determinante importante de morbilidad y mortalidad en los pacientes con LES. Un estudio realizado en Sudáfrica ha demostrado que más de la mitad de los 226 pacientes con LES de una clínica de lupus habían muerto o se ha perdido en el seguimiento a los 55 meses, y LN fue el único factor significativo asociado con la mortalidad en el análisis multivariado, con una tasa de supervivencia a 5 años del 60\% (Wadee, Tikly, \& Hopley, 2007)

En pacientes con lupus eritematoso sistémico (LES), la nefritis lúpica es una complicación común y grave que a menudo requiere una terapia inmunosupresora agresiva. Los resultados de los ensayos controlados aleatorios documentan que la mayoría de los pacientes con nefritis lúpica grave logran una remisión completa o parcial después de 6 meses de terapia de inducción con regímenes basados en micofenolato mofetilo o ciclofosfamida. Sin embargo, los brotes renales todavía pueden ocurrir durante la terapia de mantenimiento y contribuyen a la morbilidad en pacientes con LES (Mosca, 2002). La incidencia informada de brotes renales en 


\section{Diagnóstico y tratamiento de la nefritis lúpica}

Vol. 3, núm. 3., (2019)

María Soledad Novillo Valdivieso; Irina María Ayala López; Marlith Gabriela Mora Gonzalez; Leandro Olivero Hurtado Herdoiza

grandes cohortes de ensayo clínico de pacientes con nefritis lúpica varía sustancialmente, tanto como consecuencia de diferentes definiciones de brotes y debido a la inclusión de poblaciones heterogéneas de pacientes tratados con diferentes regímenes y seguimiento. El uso prolongado de la terapia de mantenimiento y la monitorización cuidadosa han ayudado a disminuir la incidencia y la gravedad de los brotes renales en pacientes con LES durante los últimos años. Si la medición de nuevos biomarcadores de lesión renal reducirá aún más la incidencia y la gravedad de brotes renales en pacientes con nefritis lúpica aún no se ha demostrado; sin embargo, la amplia aceptación de definiciones nuevas y precisas, como las de la Liga Europea contra el Reumatismo (EULAR), podría ayudar en el estudio de los brotes renales en el futuro. Dichas definiciones también podrían facilitar el desarrollo de tratamientos más efectivos y menos tóxicos para los brotes renales que los que se usan actualmente. En esta revisión, discutimos las definiciones de los brotes renales y describimos los avances en el diagnóstico y el tratamiento de los brotes renales.

\section{Metodología.}

Para el desarrollo de este proceso investigativo, se plantea como metodología la encaminada hacia una orientación científica particular que se encuentra determinada por la necesidad de indagar en forma precisa y coherente una situación, en tal sentido (Davila, 2015) define la metodología "como aquellos pasos previos que son seleccionados por el investigador para lograr resultados favorables que le ayuden a plantear nuevas ideas".(p.66) 


\section{Diagnóstico y tratamiento de la nefritis lúpica}

Vol. 3, núm. 3., (2019)

María Soledad Novillo Valdivieso; Irina María Ayala López; Marlith Gabriela Mora Gonzalez; Leandro Olivero Hurtado Herdoiza

Lo citado por el autor, lleva a entender que el desarrollo de la acción investigativa busca simplemente coordinar acciones enmarcadas en una revisión bibliográfica con el fin de complementar ideas previas relacionadas al diagnóstico y tratamiento de la nefritis lúpica a través de una revisión de literatura, para así finalmente elaborar un cuerpo de consideraciones generales que ayuden a ampliar el interés propuesto.

\section{Tipo de Investigación}

Dentro de toda práctica investigativa, se precisan acciones de carácter metodológico mediante las cuales, se logra conocer y proyectar los eventos posibles que la determinan, así como las características que hacen del acto científico un proceso interactivo ajustado a una realidad posible de ser interpretada. En este sentido, se puede decir, que la presente investigación corresponde al tipo documental, definido por Castro (2016), "se ocupa del estudio de problemas planteados a nivel teórico, la información requerida para abordarlos se encuentra básicamente en materiales impresos, audiovisuales y /o electrónicos”. (p.41).

En consideración a esta definición, la orientación metodológica permitió la oportunidad de cumplir con una serie de actividades inherentes a la revisión y lectura de diversos documentos donde se encontraron ideas explicitas relacionadas con los tópicos encargados de identificar a cada característica insertada en el estudio. Por lo tanto, se realizaron continuas interpretaciones con el claro propósito de revisar aquellas apreciaciones o investigaciones propuestas por diferentes investigadores relacionadas con el tema de interés, para luego dar la respectiva argumentación a los planteamientos, en función a las necesidades encontradas en la indagación.

\section{Fuentes Documentales}

Revista Científica Mundo de la Investigación y el Conocimiento. 3 (3). pp. 410-427 


\section{Diagnóstico y tratamiento de la nefritis lúpica}

Vol. 3, núm. 3., (2019)

María Soledad Novillo Valdivieso; Irina María Ayala López; Marlith Gabriela Mora Gonzalez; Leandro Olivero Hurtado Herdoiza

El análisis correspondiente a las características que predomina en el tema seleccionado, llevan a incluir diferentes fuentes documentales encargadas de darle el respectivo apoyo y en ese sentido cumplir con la valoración de los hechos a fin de generar nuevos criterios que sirven de referencia a otros procesos investigativos. Para (CASTRO, 2016) las fuentes documentales incorporadas en la investigación documental o bibliográfica, "representa la suma de materiales sistemáticos que son revisados en forma rigurosa y profunda para llegar a un análisis del fenómeno".(p.41). Por lo tanto, se procedió a cumplir con la realización de una lectura previa determinada para encontrar aquellos aspectos estrechamente vinculados con el tema, con el fin de explicar mediante un desarrollo las respectivas apreciaciones generales de importancia.

\section{Técnicas para la Recolección de la Información}

La conducción de la investigación para ser realizada en función a las particularidades que determinan a los estudios documentales, tiene como fin el desarrollo de un conjunto de acciones encargadas de llevar a la selección de técnicas estrechamente vinculadas con las características del estudio. En tal sentido, (Bolívar, 2015), refiere, que es "una técnica particular para aportar ayuda a los procedimientos de selección de las ideas primarias y secundarias”. (p. 71).

Por ello, se procedió a la utilización del subrayado, resúmenes, fichaje, como parte básica para la revisión y selección de los documentos que presentan el contenido teórico. Es decir, que mediante la aplicación de estas técnicas se pudo llegar a recoger informaciones en cuanto a la revisión bibliográfica de los diversos elementos encargados de orientar el proceso de investigación. Tal como lo expresa, (Bolívar, 2015) "las técnicas documentales proporcionan las 


\section{Diagnóstico y tratamiento de la nefritis lúpica}

Vol. 3, núm. 3., (2019)

María Soledad Novillo Valdivieso; Irina María Ayala López; Marlith Gabriela Mora Gonzalez; Leandro Olivero Hurtado Herdoiza

herramientas esenciales y determinantes para responder a los objetivos formulados y llegar a resultados efectivos" (p. 58). Es decir, para responder con eficiencia a las necesidades investigativas, se introdujeron como técnica de recolección el método inductivo, que hizo posible llevar a cabo una valoración de los hechos de forma particular para llegar a la explicación desde una visión general.

Asimismo, se emplearon las técnicas de análisis de información para la realización de la investigación que fue ejecutada bajo la dinámica de aplicar diversos elementos encargados de determinar el camino a recorrer por el estudio, según, (Bolívar, 2015) las técnicas de procesamiento de datos en los estudios documentales "son las encargadas de ofrecer al investigador la visión o pasos que debe cumplir durante su ejercicio, cada una de ellas debe estar en correspondencia con el nivel a emplear" (p. 123). Esto indica, que para llevar a cabo el procesamiento de los datos obtenidos una vez aplicado las técnicas seleccionadas, tales como: fichas de resumen, textual, registros descriptivos entre otros, los mismos se deben ajustar al nivel que ha sido seleccionado.

\section{Resultados.}

\section{Clasificación}

La clasificación de los LN se basa en características histológicas, utilizando la Sociedad Internacional de Nefrología (ISN) y los criterios Renal Pathology Society (RPS) desarrollada en 


\section{Diagnóstico y tratamiento de la nefritis lúpica}

Vol. 3, núm. 3., (2019)

María Soledad Novillo Valdivieso; Irina María Ayala López; Marlith Gabriela Mora Gonzalez; Leandro Olivero Hurtado Herdoiza

2003 (Tabla 1) (Wadee, Tikly, \& Hopley, Causes and predictors of death in South Africans with systemic lupus erythematosus, 2007). Aunque la clasificación es principalmente glomerulocentrica, incluye características de la enfermedad tubulointersticial, de la que dispone de cronicidad puede ser determinada. La relación entre la clase histológica de LN y el curso clínico de la enfermedad es bien reconocido. Los pacientes con clase II y clase V (LN membranosa pura) enfermedad que por lo general tiene una lenta disminución de la función renal durante largos períodos de observación. En contraste, los pacientes con clase III y clase IV (o aquellos con clase $\mathrm{V}$ mezclado) la enfermedad en su mayoría tiene un curso más agresivo de la enfermedad. Diversos estudios han demostrado que las formas proliferativas de LN (es decir, de clase V mixto de clase III, y de clase IV) se producen con más frecuencia que las otras morfologías histológicas.

\section{Diagnóstico}

Uno de los principales retos en relación con el LES es su detección precoz y el diagnóstico. Muchos médicos asumen que el LES es una enfermedad poco frecuente por lo tanto, rara vez se considera como un diagnóstico diferencial, excepto cuando los pacientes presentan características clásicas, tales como eritema malar y dolor en las articulaciones inflamadas de las manos y los pies. Por lo tanto, muchos pacientes tienden a permanecer enfermo durante períodos prolongados antes de que se haga el diagnóstico. En algunos casos, daño crónico a órganos como los riñones se habría producido.

Papel de los análisis de orina y la microscopia de orina 


\section{Diagnóstico y tratamiento de la nefritis lúpica}

Vol. 3, núm. 3., (2019)

María Soledad Novillo Valdivieso; Irina María Ayala López; Marlith Gabriela Mora Gonzalez; Leandro Olivero Hurtado Herdoiza

LN es poco probable que se presente solo, a menudo se manifiesta con otras características extrarrenales, tales como dolor en las articulaciones, erupción malar, úlceras orales y fotosensibilidad. Los pacientes con LN es probable que presente con el síndrome nefrítico (oliguria, proteinuria mínima, hematuria, hipertensión y azotemia) o con características del síndrome nefrótico (anasarca, proteinuria pesada e hipoalbuminemia). Análisis de orina (varilla de nivel y el examen microscópico) presenta la mejor oportunidad para la identificación temprana de LN como es probable que muestran la presencia de sangre y proteína en la orina una tira reactiva y microscopía de orina permite la identificación de diversos desechos urinarios (glóbulos rojos, granular, hialina). LN debe sospecharse fuertemente en cualquier paciente con LES con altos títulos de ADN de doble cadena (dsDNA) y una varilla de nivel positivo para sangre. Las características de análisis de orina se han demostrado que se correlaciona con la presencia de LN clase proliferativa, por lo tanto, cada paciente con LES debe tener un análisis de orina realizado en cada visita a la clínica (Okpechi, Swanepoel, \& Tiffin, 2012)

Tabla 1. Abreviada Sociedad Internacional de Nefrología / Renal Pathology sociedad de clasificación de la nefritis lúpica (2003)

\footnotetext{
clase I Minimal lupus nefritis mesangial

clase II Mesangial lupus nefritis proliferativa

Clase III Focal lupus nefritis (<50\% participación)

Difusa segmentaria o difusa nefritis lúpica mundial

clase IV (Participación $\geq 50 \%$ )
} 


\section{Diagnóstico y tratamiento de la nefritis lúpica}

Vol. 3, núm. 3., (2019)

María Soledad Novillo Valdivieso; Irina María Ayala López; Marlith Gabriela Mora Gonzalez; Leandro Olivero Hurtado Herdoiza

Clase $\mathrm{V}$ nefritis lúpica membranosa *

clase VI esclerosante avanzada nefritis lúpica

* Puede ser puro nefritis lúpica membranosa o tipo V mixto si se combina con clase III o de clase IV.

Fuente: (Weening, D’Agati, \& Schwartz, 2004)

El papel de la biopsia renal

El diagnóstico definitivo de LN requiere una biopsia renal. La histología renal también permite clasificación de LN y ayuda en el pronóstico. Todas las pautas recomiendan una biopsia renal cuando hay una sospecha de afectación renal, como parámetros clínicos y de laboratorio, aunque útil, no puede predecir con precisión la clase histológica. La indicación para una biopsia renal en pacientes con LES incluye disminución persistente de la función renal, proteinuria $(\geq 1.0$ g / 24 h) o proteinuria ( $\geq 0.5$ g / 24 h) si está asociado con hematuria (5 glóbulos rojos (glóbulos rojos) / campo de alta potencia) y sedimento urinario activo (moldes granulares, moldes de glóbulos blancos (WBC), moldes de RBC). Se recomienda examinar la biopsia por microscopía óptica, inmunofluorescencia (o inmunohistoquímica) y donde sea posible por microscopía electrónica (Wilhelmus, Bajema, \& Bertsias, 2016). La cuantificación de índices y descripción de actividad y cronicidad de las lesiones vasculares e intersticiales también son recomendado. Se indica una biopsia renal repetida si hay evidencia de empeoramiento de la enfermedad o enfermedad refractaria al tratamiento, evidencia de recaída (para mostrar transformación o progresión en clase histológica o cambio de actividad y núcleos de cronicidad).

\section{Tratamiento}




\section{Diagnóstico y tratamiento de la nefritis lúpica}

Vol. 3, núm. 3., (2019)

María Soledad Novillo Valdivieso; Irina María Ayala López; Marlith Gabriela Mora Gonzalez; Leandro Olivero Hurtado Herdoiza

El tratamiento de LN es dictada por la clase de la enfermedad y el grado de índices de actividad y cronicidad. Todos los pacientes deben recibir terapias adyuvantes como se indica y si se tolera. No debe haber una evaluación de riesgo-beneficio al decidir si utilizar la inmunosupresión en pacientes con un aumento de los índices de cronicidad (es decir glomerular esclerosis con fibrosis tubulointersticial) Debido al aumento de riesgo de efectos secundarios de tratamiento. El enfoque para el tratamiento a menudo implica dos fases para los pacientes con LN proliferativa (clase III, clase IV y V clase mixta).

\section{La terapia de inducción}

La terapia de inducción no se utiliza para pacientes con LN Clase I, II, V (clase V puro con proteinuria en rango sub-nefrótico) y VI. El enfoque a la inducción implica el uso de 3 pulsos consecutivos de metilprednisolona por vía intravenosa (500 - $750 \mathrm{mg}$ al día) junto con otro inmunosupresor: ciclofosfamida (CYC) (terapia de pulso intravenosa oral o mensual al día) o micofenolato de mofetilo (MMF). El paciente debe continuar en la prednisona oral (1 mg / $\mathrm{kg} /$ día) después de completar el tratamiento de pulso con metilprednisolona. CYC es a menudo el fármaco de elección para muchos clínicos debido a la fácil accesibilidad y costo; sin embargo, el uso de MMF es cada vez mayor. Varios estudios han evaluado la eficacia y seguridad de CYC con MMF o placebo durante la terapia de inducción en pacientes con LN (Chan, 2015). La duración recomendada de la terapia de inducción es de 6 meses; durante este tiempo, la dosis de corticosteroides orales debe ser destetado.

\section{La terapia de mantenimiento}




\section{Diagnóstico y tratamiento de la nefritis lúpica}

Vol. 3, núm. 3., (2019)

María Soledad Novillo Valdivieso; Irina María Ayala López; Marlith Gabriela Mora Gonzalez; Leandro Olivero Hurtado Herdoiza

El tratamiento se administra durante la fase de inducción de la terapia y se des intensifica en la fase de mantenimiento. El objetivo de esta última fase es la de mantener la respuesta (remisión) adquirida durante la fase de inducción y por lo tanto para retardar la progresión de la enfermedad renal crónica. Los agentes inmunosupresores comúnmente usados incluyen MMF, azatioprina (AZA), y corticosteroides. Calcineurina bitors INHI pueden utilizarse en circunstancias especiales, como en los casos de intolerancia a MMF o AZA o en pacientes con persistente proteinuria pesada. Actualmente no existe consenso sobre la duración de la terapia de mantenimiento. No obstante, la decisión de retirar la inmunosupresión de mantenimiento debe guiarse por la respuesta clínica completa sostenida durante un período de al menos 2 años. La retirada debe hacerse de forma gradual. En resultados debilitantes o complicaciones potencialmente mortales de la inmunosupresión, la terapia debe ser retirada.

\section{Terapias complementarias}

Las terapias complementarias suelen iniciarse durante la fase de inducción del tratamiento $\mathrm{y}$, aunque algunos necesitan ser descontinuado después de completar este tratamiento, otros tendrán que ser continuado durante la fase de mantenimiento. terapias adyuvantes comúnmente recomendados en LN incluyen:

- Renina angiotensina aldosterona (RAAS) de inhibición para proteinuria y el tratamiento de la presión arterial (objetivo <130/80 mmHg).

- Protección ósea con suplementos de calcio y vitamina D. 


\section{Diagnóstico y tratamiento de la nefritis lúpica}

Vol. 3, núm. 3., (2019)

María Soledad Novillo Valdivieso; Irina María Ayala López; Marlith Gabriela Mora Gonzalez; Leandro Olivero Hurtado Herdoiza

- La cloroquina para todos los pacientes (menos que estén contraindicados, por ejemplo, alteración de la visión).

- El tratamiento de la hiperlipidemia con (lipoproteína de objetivo de baja densidad <2.6 mmol / L) estatinas.

- Acido acetilsalicílico en dosis bajas en pacientes con síndrome antifosfolípido.

- Anticoagulante para ser considerado en pacientes con síndrome nefrótico y albúmina <20 $\mathrm{g} / \mathrm{L}$.

- Evitar la vacunación con virus vivos o atenuados durante la supresión inmune.

- Tuberculosis profilaxis con isoniazida (para aquellos en regiones altamente endémicas TB).

\section{Tratamiento de LN refractario}

Menos del 50\% de los pacientes son capaces de lograr una remisión completa durante los 6 meses de terapia de inducción. Se puede tomar hasta 2 años para alcanzar la remisión en muchos pacientes. El cambio a un agente alternativo se recomienda para pacientes que no mejoran dentro de 3 - 4 meses, o que no lograron una respuesta parcial después de 6 - 12 meses, o una respuesta completa después de 2 años de tratamiento. Las opciones de tratamiento incluyen el cambio de MMF a CYC o desde CYC a MMF; rituximab puede administrarse como complemento tratamiento o monoterapia. Otras opciones incluyen el uso de inhibidores de la 


\section{Diagnóstico y tratamiento de la nefritis lúpica}

Vol. 3, núm. 3., (2019)

María Soledad Novillo Valdivieso; Irina María Ayala López; Marlith Gabriela Mora Gonzalez; Leandro Olivero Hurtado Herdoiza

calcineurina, inmunoglobulina intravenosa y el intercambio de plasma para los pacientes con glomerulonefritis rápidamente progresiva.

\section{Tratamiento de la Clase VI LN}

La inmunosupresión para pacientes clase VI debe ser dictada por manifestaciones extrarrenales de LES. Estos pacientes deben estar preparados para la terapia de reemplazo renal (hemodiálisis, diálisis peritoneal o trasplante). Tratamiento y prevención de factores de riesgo cardiovascular (por ejemplo, control de la presión sanguínea, las estatinas para la dislipidemia) deben continuarse.

Vigilancia de toxicidad de los medicamentos y actividad de la enfermedad

Se recomienda que el peso corporal, la presión sanguínea, la creatinina sérica, análisis de orina (por proteinuria y sedimento urinario), C3 / C4, anti-dsDNA, albúmina de suero y el recuento de sangre completa puede realizar en cada visita clínica. Los pacientes con nefritis activa deben ser vistos mensualmente o con mayor frecuencia, mientras que aquellos que no tienen la enfermedad activa debe ser visto dos veces al año o cada tres meses. Esto asegura el seguimiento de actividad de la enfermedad. enfoques útiles a la detección de toxicidad de los medicamentos / efectos secundarios se resumen en la Tabla 2.

Las opciones de tratamiento para pacientes embarazadas $L N$ 


\section{Diagnóstico y tratamiento de la nefritis lúpica}

Vol. 3, núm. 3., (2019)

María Soledad Novillo Valdivieso; Irina María Ayala López; Marlith Gabriela Mora Gonzalez; Leandro Olivero Hurtado Herdoiza

LN pacientes embarazadas a menudo presentan un reto para el clínico, ya que el embarazo puede ser un disparador para una mayor actividad de la enfermedad. Por lo tanto, algunas directrices recomiendan que el embarazo no debe ser planificado hasta la remisión se ha alcanzado y mantenido durante al menos 6 meses. Las pacientes embarazadas con LN tienen un mayor riesgo de preeclampsia, parto prematuro y la pérdida fetal. Por consiguiente, deben ser manejados por un equipo multidisciplinario.

Tabla 2. Los efectos adversos de los fármacos y el enfoque de vigilancia en pacientes con nefritis lúpica

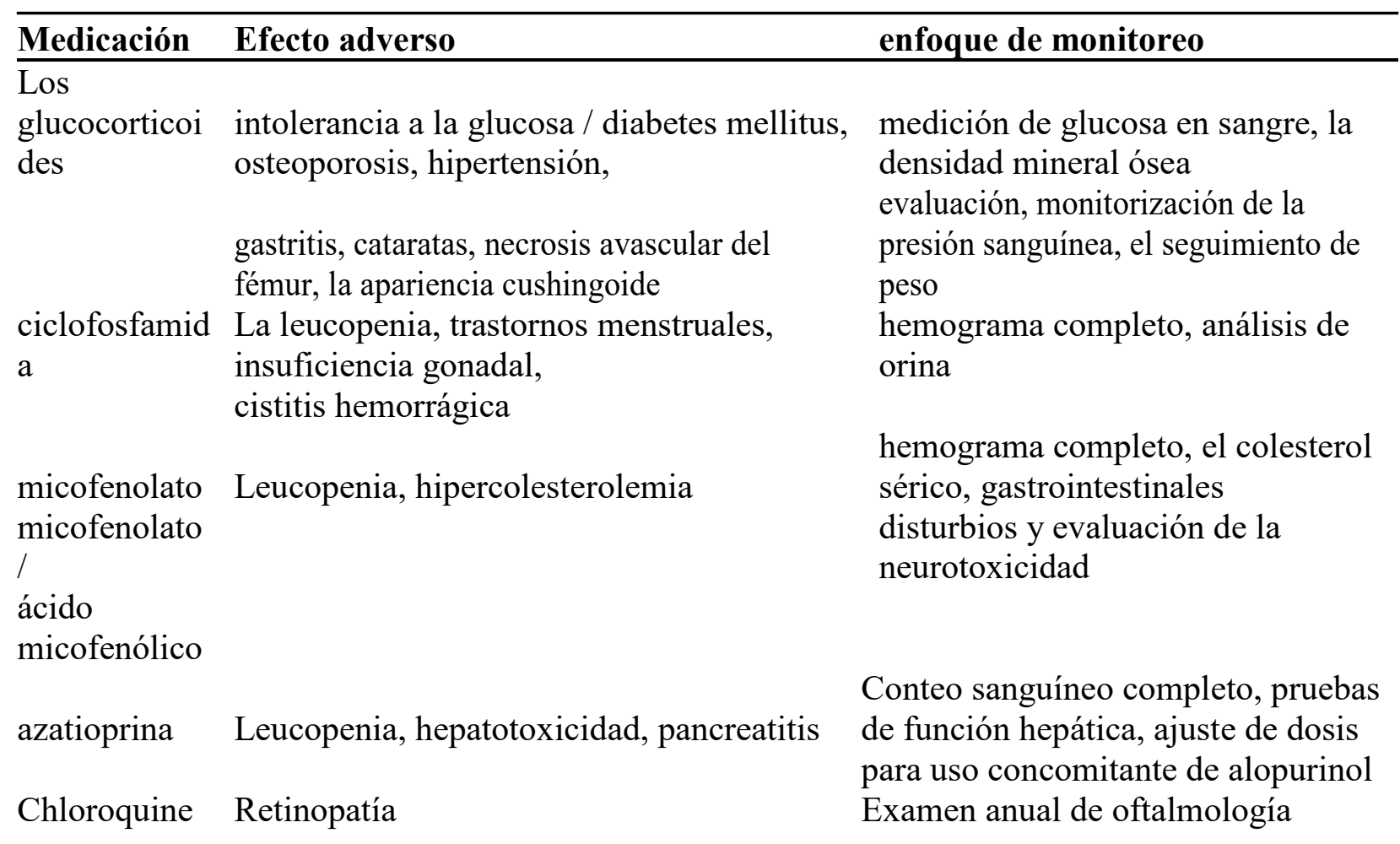

\section{Conclusiones.}




\section{Diagnóstico y tratamiento de la nefritis lúpica}

Vol. 3, núm. 3., (2019)

María Soledad Novillo Valdivieso; Irina María Ayala López; Marlith Gabriela Mora Gonzalez; Leandro Olivero Hurtado Herdoiza

Los brotes renales en pacientes con nefritis lúpica están asociados con la supervivencia renal alterada. Es necesaria una estrecha vigilancia de los cambios en el sedimento urinario, los aumentos en la proteinuria y los cambios en los marcadores serológicos, como los anticuerpos contra el dsDNA, para permitir el reconocimiento temprano y el tratamiento oportuno de los brotes renales (en particular los brotes de proteínas urinarias). Dicha monitorización es especialmente importante en el período posterior a la inducción inicial de la remisión y durante la disminución o retirada de la terapia inmunosupresora. Los datos sobre los regímenes de tratamiento óptimos son actualmente limitados y estudios multicéntricos prospectivos que comparan ciclofosfamida intravenosa con micofenolato mofetilo oral y evaluar rituximab y otros agentes biológicos son necesarios.

Para prevenir los brotes renales, la terapia de mantenimiento con dosis adecuadas debe administrarse durante un período prolongado de tiempo. La duración óptima de la terapia de mantenimiento aún no se ha determinado, pero para la mayoría de los pacientes con nefritis lúpica grave, es probable que sea necesaria una terapia de mantenimiento durante al menos 3-4 años. Para reducir el riesgo de posibles efectos adversos del tratamiento inmunosupresor a largo plazo, proponemos que las dosis utilizadas para la terapia de mantenimiento se reduzcan lentamente después de este período de tiempo en pacientes que han alcanzado la remisión completa. También son necesarios más estudios para determinar la combinación óptima de marcadores para monitorear la actividad de la enfermedad y diagnosticar brotes renales sin depender de biopsias secuenciales. Monitoreo cuidadoso de los pacientes en remisión, experiencia de los médicos y una buena relación paciente-médico para reducir la falta de adherencia a La terapia es un requisito previo para obtener una supervivencia y función renal 


\section{Diagnóstico y tratamiento de la nefritis lúpica}

Vol. 3, núm. 3., (2019)

María Soledad Novillo Valdivieso; Irina María Ayala López; Marlith Gabriela Mora Gonzalez; Leandro Olivero Hurtado Herdoiza

óptimas en pacientes con nefritis lúpica. Se espera que, con un tratamiento más temprano y efectivo de pacientes con brotes renales, la morbilidad y mortalidad asociadas con la nefritis lúpica se reduzcan aún más.

\section{Bibliografía.}

Bolívar, J. (2015). Investigación Documental. México. Pax.

Cameron, J. (1999). Lupus nephritis. J Am Soc Nephrol, 413-424.

Castro, J. (2016). Técnicas Documentales. México. Limusa.

Chan, T. (2015). Treatment of severe lupus nephritis: The new horizon. Nat Rev Nephrol, 46-61.

Davila, A. (2015). Concepto de terminos cientificos. Caracas: Oasis.

Mosca, M. (2002). Renal flares in 91 SLE patients with diffuse proliferative glomerulonephritis. Kidney Int. , 1502-1509.

Okpechi, I., Swanepoel, C., \& Tiffin, N. (2012). Clinicopathological insights into lupus nephritis in South Africans: A study of 251 patients. Lupus, 1017-1024.

Wadee, S., Tikly, M., \& Hopley, M. (2007). Causes and predictors of death in South Africans with systemic lupus erythematosus. Rheumatology, 1487-1491. Obtenido de [http://dx.doi.org/10.1093/ rheumatology/kem180

Wadee, S., Tikly, M., \& Hopley, M. (2007). Causes and predictors of death in South Africans with systemic lupus erythematosus. Rheumatology, 1487-1491.

Weening, J., D’Agati, V., \& Schwartz, M. (2004). The classification of glomerulonephritis in systemic lupus erythematosus revisited. J Am Soc Nephrol, 241-250.

Wilhelmus, S., Bajema, I., \& Bertsias, G. (2016). Lupus nephritis management guidelines compared. Nephrology Dialysis Transplantation, 31(6), 904-913. 